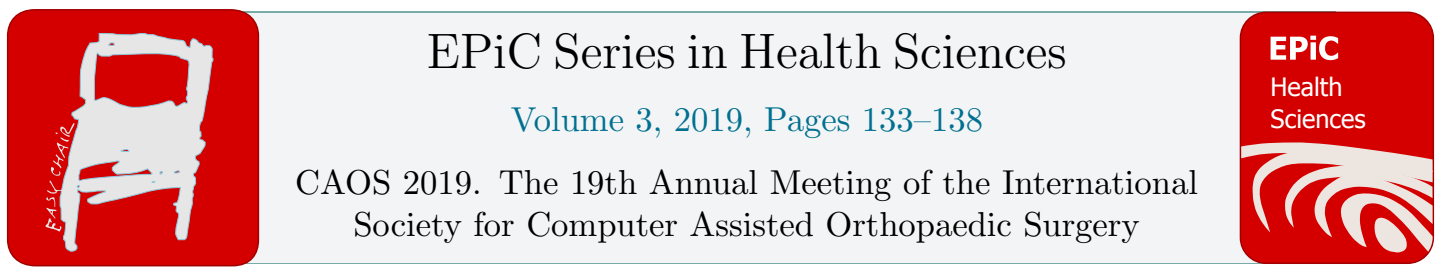

\title{
Anterior Mechanical Navigation Device is as Accurate as Lateral Device for Hip Socket Position
}

\author{
David Freccero $\mathrm{MD}^{1^{*}}$, Justin Koh MD, MA ${ }^{1}$, Jeansol Kang MD ${ }^{1}$, Chris \\ Fang $\mathrm{BA}^{2}$ and Eric Smith $\mathrm{MD}^{1}$ \\ ${ }^{1}$ Department of Orthopaedic Surgery, Boston Medical Center, Boston, MA \\ ${ }^{2}$ Boston University School of Medicine, Boston, MA \\ david.freccero@bmc.org
}

\begin{abstract}
Objectives: In total hip arthroplasty (THA), accurate acetabular component position promotes prosthetic hip joint stability and longevity, and minimizes polyethylene wear. Image-based mechanical navigation is known to improve accuracy and reproducibility of accurate cup position intraoperatively via the posterior approach and the superior capsular approach. The purpose of this study was to assess the accuracy of acetabular component position using image-based mechanical navigation via the direct anterior approach (DAA). Methods: We prospectively followed 96 patients who underwent THA with one fellowship-trained arthroplasty surgeon over a nine-month period. Thirty-three patients underwent DAA THA with the anterior HipXpert device (Group 1), and 63 patients underwent posterior approach THA with the lateral HipXpert mechanical navigation device, serving as an operative control group (Group 2). Standard postoperative plain film radiographic measurements of acetabular component inclination and anteversion were assessed. Results: The average inclination angle was 38.6 degrees and 40.6 degrees in Groups 1 and 2, respectively. The average anteversion angle was 27.6 degrees and 30.1 degrees in Groups 1 and 2, respectively. There were no postoperative hip dislocations and no study patients underwent revision THA at an average follow-up of 12 months. There were no patient outliers in Groups I or II with inclination angles or anteversion angles outside 10 degrees of the preoperatively planned values. Conclusion: The anterior HipXpert mechanical navigation device enhances accurate acetabular component position and may reduce outlier component placement. Acetabular socket position is as accurate using the anterior device as it is using the lateral device.
\end{abstract}




\section{Introduction}

Acetabular component position in total hip arthroplasty (THA) is critical to optimize hip biomechanics, reduce impingement and instability, minimize wearinduced osteolysis, and promote implant longevity. The first concept of a safe zone for acetabular cup positioning was introduced by Lewinnek et al. in 1978 and has been widely used to guide assessment of component placement [1]. Recently, however, the clinical utility of this safe zone has come into question [2]. As pelvic tilt and spinopelvic attitude and flexibility vary between patients, the functional orientation of the acetabular component - and therefore the optimal socket position is likely to be patient-specific, rather than in a fixed zone. Thus, optimizing acetabular component positioning may require individualized pre-operative planning.

Intra-operative technology assistance has been increasingly used to improve accuracy and precision of component placement in THA procedures, with a recent estimated overall use of $1.9 \%$ and a nearly tenfold increase from 2008 to 2015 [3]. Smart mechanical navigation devices such as the HipSextant and HipXpert systems (Surgical Planning Associates Inc., Boston, MA) have been shown to provide reliable and accurate acetabular cup position in THA [4-6]. Although studies have looked at these devices using a posterior approach, no such studies have evaluated the anterior device using the anterior approach. In this study, we sought to assess the accuracy of acetabular component position using an image-based mechanical navigation device via the direct anterior approach (DAA).

\section{Materials and Methods}

All patients who underwent primary THA using either the anterior or lateral HipXpert mechanical navigation device (Surgical Planning Associates Inc., Boston, MA) with one fellowship-trained arthroplasty surgeon at a single academic center were prospectively followed over a nine-month period (December 2016 to September 2017). Patients who underwent DAA THA with the anterior HipXpert device were included in the study group, Group 1. Group 2 represented an operative control group and included patients who underwent THA using the posterior approach with the lateral HipXpert mechanical navigation device.

The HipXpert device is an adjustable and reusable frame that is applied to specific points on the pelvis during surgery to assist with socket position. It is placed on the hemipelvis, with the base point behind the posterior wall of the acetabulum, $20 \mathrm{~mm}$ from the infracotyloid notch. The second point is on the lateral ilium, adjacent to the anterior superior iliac spine, and the third point is equidistant from the other two points on the ilium, anterior to the sciatic notch. A virtual pelvis model is created using preoperative CT scans, and an ideal socket position is formulated on the model. 
Preoperative and postoperative standing AP radiographs were obtained using a standardized protocol. The anterior pelvic plane and operative anteversion were used to plan the acetabular component position. There was no significant difference in preoperative planned values between groups. Radiographic measurements of acetabular component inclination and anteversion were made using TraumaCad digital software on standard standing postoperative AP pelvis radiographs. Patient demographics, including age, gender, and surgical procedure, were also recorded.

AP pelvic radiographs were measured to calculate the postoperative cup inclination and version. The average value was then compared to the preoperatively planned values. An outlier was defined as outside of a range of $\pm 10^{\circ}$ of inclination and/or anteversion from the planned preoperative orientation.

Statistical analysis included student $t$ tests to determine if the mean values of the absolute error for anteversion and inclination were less than $10^{\circ}$. A p value less than 0.05 was considered statistically significant.

\section{Results}

Ninety-five patients (96 hips) were included in the study. The study group (Group 1) was comprised of 32 patients (33 hips), and the surgical control group (Group 2) was comprised of 63 patients (63 hips).

There were no significant differences in age, BMI, or gender between the two groups. The average acetabular inclination angle was $38.6^{\circ}$ and $40.6^{\circ}$ in Groups 1 and 2 , respectively. The average acetabular anteversion angle was $27.6^{\circ}$ and $30.1^{\circ}$ in Groups 1 and 2, respectively. The target cup inclination and anteversion were achieved within $10^{\circ}$, simultaneously, in all cases using the anterior HipXpert and lateral HipXpert device. There were no intraoperative complications related to the anterior or lateral device. At an average follow-up of 12 months, there were no prosthetic hip joint dislocations and no patient required revision. 


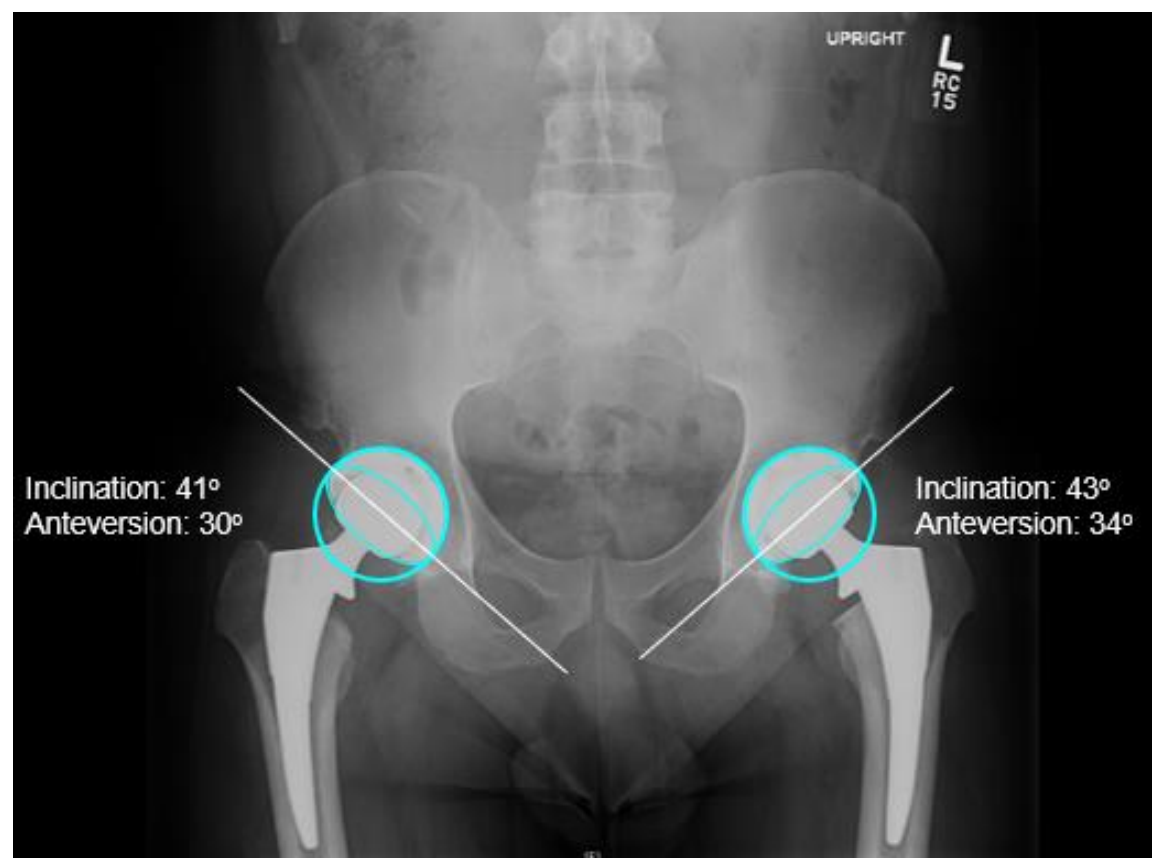

Fig. 1. Postoperative AP radiograph of a patient who underwent staged bilateral DAA THA for bilateral hip osteoarthritis, shown with acetabular inclination and anteversion measurements.

\section{Discussion}

Acetabular component malposition can result in mechanical complications, including dislocation, impingement, increased wear, and increased revision rates. Two recent studies revealed poor accuracy of socket orientation with free-hand insertion techniques [7-8]. Despite this, use of computer-assisted navigational systems among arthroplasty surgeons remains relatively uncommon and few studies have assessed the accuracy of these devices. Jennings et al. evaluated 47 hips that underwent primary THA using the lateral HipSextant mechanical navigation device via the posterior approach and found that $100 \%$ of acetabular components were within $10^{\circ}$ of the planned anteversion and inclination targets [4]. Similarly, Murphy et al. reviewed postoperative CT studies of 37 hips that underwent primary THA using the posterior approach and lateral HipXpert device and also found no outliers in either anteversion or inclination, demonstrating the accuracy and precision of the device [5].

Limitations of this study include the non-randomized nature, which introduces the possibility of patient selection bias. All procedures were performed by a single surgeon, which limits the generalizability of the results. Anteversion measurements 
were made using plain radiographs, which may be inaccurate; CT would be a better evaluation tool in further studies [9].

To our knowledge, this is the first clinical study evaluating the anterior HipXpert device using the anterior approach. Our results suggest that it is as accurate in achieving target cup orientation as the lateral device, which has been shown to increase the surgical accuracy of acetabular component orientation compared to conventional free-hand techniques. These devices may improve patient outcomes by reducing outliers and mechanical complications after THA.

\section{References}

1. Lewinnek GE, Lewis JL, Tarr R, Compere CL, Zimmerman JR. Dislocations after total hip-replacement arthroplasties. J Bone Joint Surg Am. 1978;60(2):217-220.

2. Murphy WS, Yun HH, Hayden B, Kowal JH, Murphy SB. The safe zone range for cup anteversion is narrower than for inclination in THA. Clin Orthop Relat Res. 2018;476(2):325-335.

3. Boylan M, Suchman K, Vigdorchik J, Slover J, Bosco J. Technology-assisted hip and knee arthroplasties: an analysis of utilization trends. J Arthroplasty. 2018;33(4):1019-1023.

4. Jennings JM, Randell TR, Green CL, Zheng G, Wellman SS. Independent evaluation of a mechanical hip socket navigation system in total hip arthroplasty. J Arthroplasty. 2016;31(3):658-661.

5. Murphy WS, Borchard K, Kowal JH, Murphy SB. Clinical accuracy of a smart mechanical navigation system for cup alignment as measured by postoperative CT. Orthop Proc. 2017;99-B(Suppl 20):76.

6. Steppacher SD, Kowal JH, Murphy SB. Improving cup positioning using a mechanical navigation instrument. Clin Orthop Relat Res. 2011;469(2);423428.

7. Barrack RL, Krempec JA, Clohisy JC, et al. Accuracy of acetabular component position in hip arthroplasty. J Bone Joint Surg Am. 2013;95(19):1760-1768.

8. Callanan MC, Jarrett B, Bragdon CR, et al. The John Charnley Award: risk factors for cup malpositioning: quality improvement through a joint registry at a tertiary hospital. Clin Orthop Relat Res. 2011;469(2):319-329. 
9. Manjunath KS, Soruban V, Gopalakrishna KG. Evaluation of radiological methods of assessing cup anteversion in total hip replacement. Eur J Orthop Surg Traumatol. 2015;25(8):1285-1292. 